Meta

Journal des traducteurs

Translators' Journal

\title{
Index des mots et des sujets traités dans le volume 25
}

Volume 25, numéro 4, décembre 1980

URI : https://id.erudit.org/iderudit/003206ar

DOI : https://doi.org/10.7202/003206ar

Aller au sommaire du numéro

Éditeur(s)

Les Presses de l'Université de Montréal

ISSN

0026-0452 (imprimé)

1492-1421 (numérique)

Découvrir la revue

Citer ce document

(1980). Index des mots et des sujets traités dans le volume 25. Meta, 25(4),

497-505. https://doi.org/10.7202/003206ar d'utilisation que vous pouvez consulter en ligne.

https://apropos.erudit.org/fr/usagers/politique-dutilisation/ 


\section{INDEX DES MOTS ET DES SUJETS TRAITÉS DANS LE VOLUME 25}

À (la préposition), 1, p. 91.

Abrègement, en néologie, 3, p. 345.

ACNOR, 1, p. 129.

ACNOR (normes), 1, p. 33.

Actes du Colloque international de terminologie de Baie St-Paul, 1, p. 160.

Actes du Colloque international sur la normalisation linguistique, 1, p. 160.

Actes du colloque international sur l'enseignement de la terminologie, 1, p. 161.

Adaptation au destinataire, 4, p. 399

AELE, 1, p. 129.

AFNOR, 1, p. 29.

AFNOR (Centre de documentation de l'), 1, p. 187.

AFNOR (normes), 1, p. 33.

AGRIDOQ, 1, p. 175 .

AHKMANOVA, 1, p. 155.

Aide-mémoire Technor, 1, p. 104

Ajustement (systèmes d'), 4, p. 469.

Alésages, 4 , p. 473.

Aliment en conserve, 3, p. 357.

«Aliment-service», 3, p. 357.

Aliments déshydratés, 3, p. 357.

Aliments surgelés, 3, p. 357

Aliments vite prêts, 3 , p. 358 .

Ambivalence en traduction, 4, p. 430.

Analyse de document, 1, p. 23.

Analyse d'un syntagme, 2, p. 233.

ANSI (système), 4, p. 474 .

Aphérèse et apocope, 3, p. 346.

A.P.I.Q., 2, p. 283.

Apocope en « $\mathrm{O} », 3$, p. 347.

Apocope et aphérèse, 3 , p. 346.

"Aréna,$~ 3$, p. 385.

Argot criminel et suffixation en « $\mathrm{O} », 3$, p. 350 .

Argot de la vie quotidienne et suffixation en « $\mathrm{O}$, 3, p. 351 .

Argot du monde du spectacle et suffixation en « $O », 3$, p. 351 .

Argot hospitalier et suffixation en $« O », 3$, p. 351 .

Argot militaire et suffixation en « $\mathrm{O} », 3$, p. 349.

Argot politique et suffixation en $" \mathrm{O} », 3$, p. 349 .

Argot scolaire et suffixation en $« O », 3$, p. 348 .

Argotisme en $« \mathrm{O} », 3$, p. 347.

Argotismes en $" \mathrm{O} », 3$, p. 347 .

Aristote (traductions d'), 4, p. 432.

Art de conjuguer, 1, p. 100.

ASMO, 1, p. 129.

Assistance bibliographique ponctuelle, 1, p. 25 .

Association canadienne de normalisation, 1, p. 129.

Association des traducteurs et interprètes de l’Ontario, 2, p. 281, 283.
Association européenne du libre échange, 1, p. 129.

Association française de normalisation, 1, p. 129.

Association professionnelle des Interprètes du Québec, 2, p. 282.

Atelier sur l'histoire de la traduction, 3, p. 380.

Ateliers de terminologie I et II, 3, p. 381.

Ateliers de traduction littéraire, 3, p. 382 .

A.T.I.O., 2, p. $281,283$.

A.T.I.O. (Congrès annuel de l'), 4, p. 491

Auteur-traducteur (couple), 4, p. 440

«Auto-», 2, p. 251.

Autoriser, 1, p. 90.

BADADUQ, 1, p. 174

Banque de terminologie du Québec, 1, p. 132.

Banque d'information documentaire, 1, p. 175

Banques de terminologie au Canada, 1, p. 14.

Benjamin, Walter (théorie de), 4, p. 435.

BIBLIOCOM, 1, p. 177.

Bibliographie de la France, 1, p. 64.

Bibliographie internationale des dictionnaires techniques monolingues et multilingues, 1, p. 64 .

Bibliographie sélective du traducteur (techniques), 1, p. 106.

Bibliographies (établissement de), 1, p. 25 .

Bibliothécaire (fonction d'un), 1, p. 42, 50

Bibliothèque Dag Hammarskjold, 1, p. 64 .

Bibliothèque de la Direction générale des services de traduction, 1, p. 54.

Bibliothèque de la Division de Montréal, 1, p. 55.

Bibliothèque de la division de Québec, 1, p. 55 .

"Bibliothèque de réseau», $1, \mathrm{p}, 51$.

«Bibliothèque de section», 1, p. 51

Bibliothèques (liaisons entre les), 1, p. 35 .

BNQ, 1, p. 129.

Bocal, p. 4, p. 450.

Boèce (doctrine de), 4, p. 432

Bon Usage (le), 1, p. 98.

«Boom», 2, p. 273.

Britanniques (terminologues), 1, p. 159

BSI, 1, p. 129.

Bulletin d'information produit par le docum mentaliste, 1 , p. 35 .

Bulletins signalétiques, 1, p. 175.

«Bulletins signalétiques» du CNRS, 1, p. 69.

Bureau de normalisation du Québec, 1, p. 129.

Bureau des traductions, Ottawa, 1, p. 49.

Canadian Selective Dissemination of Information, 1, p. 179.

CAN/MARC (Bibliothèque nationale du Canada), 1, p. 52 . 
CAN/OLE, 1, p. 54, 180.

CANOLE (système), 1, p. 14.

CAN/SDI, 1, p. 54, 179.

Caplygin (S.A.), 1, p. 156.

Capsuline, 1, p. 105.

Catalogue des documents, 1, p. 23.

Catalogue collectif, 1, p. 51 .

«Catalogue international des périodiques»,

Catalogueur, 1, p. 43.

CCN, 1, p. 130.

CEE, 1, p. 129.

CEI, 1, p. 129.

CEN, 1, p. 129.

Centre de documentation, 1, p. 45.

Centre de documentation, 1, p. 6.

Centre de documentation de la Direction générale de la terminologie et de la documentation, 1 , p. 56 .

Centre de documentation (schéma de fonctionnement), 1, p. 18.

Centre international de l'UNISIST, 1, p. 204.

Centre international d'information pour la terminologie, 1, p. 197.

Centres de documentation spécialisés, 1, p. 171.

Certitude, 1, p. 89.

CETMF, 1, p. 132.

CETTF, 1, p. 132.

Chaîne documentaire, 1, p. 49, 84 .

Champs lexicaux, 3, p. 312.

Champs terminologiques, 3, p. 312.

CIL, 1, p. 194.

CILF, 1, p. $131 ; 2$, p. 274

Classement de la documentation, 1, p. 15.

Classement des systèmes juridiques; le système anglo-américain et les systèmes romanogermaniques, 1, p. 147.

Classement raisonné, 1, p. 17.

Classification (systèmes de), 1, p. 31 .

Classification décimale universelle, 1, p. 85.

Classification de la documentation, 1, p. 15.

Classification des documents, 1, p. 23.

Classifications, 1, p. 85.

Classifications encyclopédiques générales, 1 , p. 85.

Clé d'accès à une banque de données, 1, p. 184.

CNOMO, 1, p. 131.

Code civil, 1, p. 140 .

COLING, 2, p. 283.

Colloque, collège Glendon, 4 , p. 391 .

Colloque international sur la traduction, 3, p. 378.

Colloque «La théorie au service de la pratique $», 3$, p. 378 .

Colloque OLF-STQ (troisième), 2, p. 278.

Colloque sur les français régionaux, 2, p. 274.

Comité consultatif des normes asiatiques, 1, p. 129.

Comité de régie du Bureau des traductions, 1, p. 57.

Comité d'étude des termes médicaux français, 1, p. 132 .
Comité d'étude des termes techniques français, 1, p. 132.

Comité européen de normalisation, 1, p. 129.

Comité technique ISO/TC 37, 1, p. 197.

Comité technique n 37 de l'ISO, 1, p. 159.

Commission de normalisation de l'outillage et de la machine-outil, 1, p. 131.

Commission de terminologie de l'Office de la langue française, $1, \mathrm{p} .131$.

Commission électrotechnique internationale, 1, p. 129.

Commission permanente de standardisation, 1, p. 130.

Communauté économique européenne, 1, p. 129.

Communications scientifiques ou techniques, 1 , p. 152.

Commutation et segmentation, 2, p. 270.

Comparaison des sens et comparaison des signifiés, 2, p. 268.

Compétence et performance en traduction, 4 , p. 438.

Compléments cognitifs, 4, p. 406.

Composé intermétallique, 1, p. 106.

Compréhension et sémantique, 2, p. 230.

Conférence intergouvernementale sur la planification des infrastructures nationales en matière de documentation, de Congrès APFUC, 3, p. 379.

Congrès de l'Association des professeurs de français des universités canadiennes, 3, p. 379.

Connaître les nuances de sa langue maternelle, 4 , p. 394.

Connotation, 4, p. 441.

Connotation comique ou péjorative de la suffixation en $\ll O *, 3$, p. 352 .

Conseil canadien des normes, 1, p. 129.

Conseil des traducteurs et interprètes du Canada, 2, p. 283.

Conseil international de la langue française, 1 , p. $131: 2$, p. 274.

Conservateur, 1, p. 45.

Conservation, 3, p. 359.

Coran (intraduisibilité du), 4, p. 441.

Corporation des traducteurs professionnels du Nouveau-Brunswick, 2, p. 283.

Calque, 4, p. 449.

Corpus d'analyse, 1 , p. 54

Corpus de référence, 1, p. 64 .

Coût moyen de la traduction, 3, p. 383.

Coutume, 1, p. 138.

CPS, 1, p. 130.

Création lexicale en français, 1, p. 91.

CST, 1, p. 152.

CTIC, 2, p. 283

«Cyclo-», 2, p. 251.

Dahlberg (I.), 1, p. 155.

Darilenko, 1, p. 155.

DATAPAC (réseau), 1, p. 175.

Datum (système), 1, p. 14.

DD, 1 , p. 56. 
«Débuter une séance», 1, p. 94.

Définition (la - en terminologie), 1, p. 156.

Département des conférences de l'ONU, 1 , p. 59.

Dépouillement de périodiques par le traducteur, 1, p. 37.

Dépouillement documentaire/dépouillement terminologique, $1, \mathrm{p}, 81$.

Descripteurs en documentation (les), 1, p. 16.

Désignation du signe, 2, p. 269.

Désinences, 1, p. 82.

D.F.C., 1, p. 94.

Dégroupement et regroupement, 1, p. 94.

DGST (Ottawa), 1, p. 54.

DGTD, 1, p. 56.

«Dicible», 2, p. 268.

Dictionnaire alphabétique et analogique de la langue française, 1, p. 88 .

Dictionnaire analogique (le), 1, p. 97.

Dictionnaire anglais-français des termes relarifs à l'électrotechnique, à l'électronique et aux applications connexes, 1, p. 109.

Dictionnaire de l'Académie (le), 1, p. 87.

Dictionnaire de l'Académie française, 1, p. 97.

Dictionnaire de la langue du 19 e et du $20^{\mathrm{e}}$ siècle (1789-1960), 1, p. 91.

Dictionnaire de l'informatique, de Bureau, 1, p. 107.

Dictionnaire des difficultés de la langue francaise, 1, p. 99.

Dictionnaire des difficultés grammaticales et lexicologiques, 1, p. 99

Dictionnaire des fabrications mécaniques, 1 , p. 108 .

Dictionnaire des huit mille verbes usuels, 1, p. 100 .

Dictionnaire des jdées suggérées par les mots, 1, p. 97.

Dictionnaire des sciences et techniques nucléaires, 1 , p. 107.

Dictionnaire des synonymes, de Bailly, 1, p. 97.

Dictionnaire des synonymes, de Bénac, 1, p. 97.

Dictionnaire des techniques (focus international), 1, p. 102.

Dictionnaire des termes utilisés dans l'industrie clu pétrole, 1, p. 109.

Dictionnaire d'informatique, de Le Garff, 1, p. 107.

Dictionnaire du français contemporain, 1, p. 93

Dictionnaire encyclopédique Quillet, 1, p. 95.

Dictionnaire étymologique de la langue francaise, 1, p. 97

Dictionnaire général de la langue française, 1, p. 88.

Dictionnaire multilingue de la machine-outil, 1, p. 108 .

Dictionnaire technique général anglais-français, 1, p. 109.

Dictionnaires (lacunes des), 2, p. 255.

Dictionnaires analogiques, 1, p. 97

Dictionnaires de langue, 1, p. 87.

Dictionnaires de synonymes, 1, p. 97.
Dictionnaires d'informatique anglais-français et français-anglais, de Ginguay, 1, p. 107.

Dictionnaires encyclopédiques, 1, p. $87 ; 95$.

Dictionnaires étymologiques, 1, p. 97.

Dictionnaires polyglottes, 2, p. 215.

Dictionnaires spécifiques, 1, p. 97.

DIC/VOC, 1, p. 53.

Didacticiel, 3, p. 294.

Diffusion sélective de l'information, 1, p. 53.

Diffusion sélective de l'information au Canada, 1, p. 197.

«Digest» d'un texte de périodique, 1, p. 76 .

Digitaliser, 1, p. 101.

Digitaliseur, 1, p. 105 .

Direction de la Documentation, Ottawa, 1, p. $50 ; 56$.

Direction générale de la terminologie et de la documentation (Ottawa), 1, p. 56.

Direction générale des services de traduction (DGST), 1, p. 54 .

Discours, 3, p. 311.

Disposable, 3 , p. 385 .

Divergence et marge, 4, 453 .

Division de la coordination du réseau de bibliothèques (Ottawa), 1, p. 54.

Division des services publics (Ottawa), 1, p. 53.

Division des services techniques (Ottawa), 1, p. 52 .

Division de traduction de l'ONU, 1, p. 60 .

Doctrine, 1, p. 138.

Doctrine et théorie de la traduction, 4, 402.

Doctrines linguistiques de la traduction, 4, 404.

Documentaliste (définition du), 1, p. 45.

Documentalistes et traducteurs (collaboration entre), 1, p. 30.

Documentation (accès à la), 1, p. 14.

Documentation (collection de la), 1, p. 23.

Documentation (documents fondamentaux), 1, p. 6 .

Documentation (évaluation de la), 1, p. 16, 65 .

Documentation (gestion de la), 1, p. 11; 26.

Documentation (information juridique), 1, p. 14

Documentation (la - en terminologie), 1, p. 156.

Documentation (objectifs de la), 1, p. $10 ; 28$.

Documentation (organisation de la), 1, p. 6.

Documentation (qualité de la), 1, p. 15.

Documentation (qualité linguistique de la), 1, p. 28.

Documentation (rentabilité), 1, p. 9; 19.

Documentation (typologie de la), 1, p. 154

Documentation automatisée, 1, p. 176.

Documentation au Canada (historique de la), 1 , p. 21 .

Documentation du traducteur juridique, 1 , p. 144.

Documentation du traducteur technique, 1 , p. 101 .

Documentation en matière de langue française, 1, p. 87.

Documentation et «langues de spécialité », 1 , p. 8 . 
Documentation et polyvalence langagière, 1, p. 11.

Documentation et production, 1 , p. 1; $9 ; 19$.

Documentation et terminologie, 1, p. 152.

Documentation et services linguistiques, 1, p. $7 ; 40$.

Documentation et traduction technique, 1, p. 7 .

Documentation juridique, 1, p. 134.

Documentation paraterminologique, 1, p. 183

Documentation terminologique, 1, p. 183.

Documentation terminologique (tableaux), 1, p. 153,166 .

Documents épars, 1, p. 71.

Donneur d'ouvrage, 4, 399.

Drezerr, 1, p. 155.

Driedger, E.A., 3, p. 316.

Droit civil, 1, p. 141.

«Droit communautaire», 3, p. 372.

Droit comparé, 1, p. 147.

Droit coutumier, 1, p. 140;3, 373 .

Droit jurisprudentiel, 1, p. 141.

Droit positif, 1, p. 147.

Droit privé, 1, p. 140

Droit public, 1, p. 140

Droit romain, 1, p. 140

Drozd, 1, p. 157.

DSI, 1, p. 53.

D.T.G., 1, p. 109.

Dupré (le), 1, p. 98.

École canado-québécoise de terminologie, 1, p. 155.

École germano-autrichienne de terminologie, 1, p. 155.

École soviétique de terminologie, 1, p. 155.

École tchécoslovaque de terminologie, 1, p. 155.

Écoles de terminologie (diverses), 1, p. 155.

Écoles de traduction (les deux), 4, 437; 442 .

Écrivains et traducteurs, 4, 396.

Éléments cognitifs non linguistiques, 4, 404.

Emprunt, 4, p. 449.

Encyclopédie de l'électricité, 1, p. 103

Encyclopédie des sciences industrielles, 1, p. 103.

Encyclopédie du bon français dans l'usage contemporain, 1 , p. 98

Équivalence dynamique, 4, 431 .

Équivalence (l'importance des), 4, p. 447.

Équivalence formelle stricte, 4,432 .

Établissement du calendrier (des travaux), 3, p. 355 .

EURODICAUTOM, 1, p. 82.

Étymologie et traduction, 4 , p. 492.

«Exégèse », 2, p. 268

Explicite et implicite de la communication, 4 , 407.

« Explosion documentaire », 1, p. 43.

Fédération internationale des traducteurs, 2, p. 283.

Felber (H.), 1, p. 155.

Féminin des noms de profession, 2, p. 249.
Fiche auteur, 1, p. 79.

Fiche phraséologique, 2, p. 236.

Fiche syntagmatique bipartite, 2, p. 235.

Fiche syntagmatique quadripartite, 2, p. 235.

Fiche syntagmatique tripartite, 2, p. 235.

Fiche terminologique (traitement documentaire de la), 1, p. 80.

Fiche titre, 1, p. $43 ; 79$.

Fiches bibliographiques, 1, p. 79 .

Fiches d'analyse, 1, p. 77.

Fiches-sujets, 1, p. 24; 43.

Fiches syntagmatiques, 2, p. 232.

Fiches unitermes, 1, p. 76.

Fichier de lecteurs, 1, p. 85.

Fichier des mots clés, 1, p. 76; 78.

Fichier des périodiques, 1, 73 .

Fichier MARC/Québécois (Bibliothèque nationale du Québec), 1, p. 52.

Fichier topographique, 1, p. 23.

Fichiers-sources, 1, 52.

FIT, 2, p. 283.

Fixation des délais d'exécution, 3, p. 355

Focus, Dictionnaire des techniques (le), 1, p. 102.

Fonction identificatrice, 4, p. 391.

Fonction laudative, 4, p. 391.

Fonction ludique, 4, p. 391.

Fonction mnémotechnique, 4, p. 391.

Fonction normative de la documentation juridique, 1, p. 140.

Français (documentation en matière de), 1, p. 87.

Français (terminologues), 1, p. 159.

Français correct (le), 1, p. 99.

Français régionaux, 2, p. 274.

FRANCIS, 1, p. 175.

Furet (le), 1, p. 106.

Germano-autrichiens (terminologues), 1, p. 155.

G.L.E., 1, 95.

G.L.L.F., 1, p. 89.

Glose, 2, p. 268.

Golovin, 1, p. 155

Grammaires et ouvrages similaires, 1, p. 98.

Grand Larousse de la langue française, 1, p. 89.

Grand Larousse encyclopédique, 1, p. 95.

Grand Robert (le), 1, p. 88.

Grevisse (le), 1, p. 99.

Grevisse, Maurice, 4, p. 491

Guide mondial des activités terminologiques, 1 , p. 204.

Guilbert (Louis), 1, p. 159.

Hausa, 4, p. 464.

Haut comité de la langue française, 1, p. 131.

Havranek, 1, p. 157.

HISCABEQ, 1, p. 177.

«Huile à chauffage $», 2$, p. 263.

Huile de chaufface, 2, p. 263.

Huile lourde, 2, p. 263.

Hypotaxe, 4, p. 434

ICIST, 1, 54, p. 178.

Idiolecte et pluralisme de l'usage, 4, p. 397. 
IEC, 1, p. 181.

Implicite et explicite de la communication, 4, p. 407.

«Index alphabétique» 1, p. 76.

Indexation en vocabulaire libre, 1, p. 75

Indexation et établissement des fichiers de documentation, 1, p. 23.

Indexation par mot clé, 1, p. 75 .

Index des documents des Nations unies, 1, p. 64 .

Informatech France-Québec, 1, p. 69, 174.

INFORMATECH-SABINE (système), 1, p. 14.

Information (communication de l'), 1, p. 47 .

Information brute (secteur $\mathrm{d}^{\prime}$ ), $1, \mathrm{p} .15$.

Information (détection de l') 1, p. 47.

Information (évaluation de l'), 1, p. 47

Information raffinée (secteur $\mathrm{d}^{\prime}$ ), 1, p. 14.

INFOTERM, 1, p. 132, 196.

Infoterm (publications d'), 1, p. 198.

ISO (système), 4, p. 472 .

Institut autrichien de normalisation, 1, p. 197.

Institut canadien de l'Information scientifique et technique, 1, p. 178.

Institut canadien pour l'information scientifique et technique, 1, p. 54 .

Interlinéaire et juxtalinéaire, 4, p. 436.

Interrogation en direct au Canada, 1, p. 180.

"Intervention à ciel ouvert », 4, p. 469

Intervention sanglante, $4, \mathrm{p}, 469$.

Inuit (substantif pluriel et adjectif), 2, p. 282.

Inuk (substantif singulier), 2, p. 282.

Inuktitut, 2, p. 282.

ISA, 1 , p. 129

ISO, 1 , p. $56,128$.

ISO (Comité technique $\mathrm{n}^{\circ} 37$ de l'), 1, p. 159

ISO R9, 2, p. 223.

ISONET, 1, p. 132, 188, 195.

Jetable, 3, p. 385.

Journalisme (glossaire bilingue des termes de), 2 , p. 273.

Judaité et traduction, 4 , p. $437,442$.

Jurisprudence, 1, p. 137

JURIVOC, 1 , p. 149.

Juxtalinéaire, 4, p. 434

Justalinéaire et interlinéaire, 4, p. 436.

Kandelaki, 1, p. 156.

Klimovitski, 1, p. 156.

Kocourek, 1, p. 157.

«Krisis der cupaischen Kultur», 4, p. 434

Kulebakin, 1, p. 156

Kutina, 1, p. 156.

KWIC (listes), 3, p. 290.

Lacunes des dictionnaires, 2, p. 255.

Langage enfantin et suffixation en $« \mathrm{O} », 3$, p. 352 .

Langage médiatique, 3 , p. 288

Langage PLATON, 3, p. 288.

Langages, 3 , p. 311

Langages à structure combinatoire, 1, p. 85 .

Langages à structure hiérarchique, 1, p. 85 .

Langages documentaires, 1, p. 85 .
Langue et parole en traduction, 4, p. 438.

Langues officielles à l'ONU, 1, p. 58 .

"L'Anglais juridique et le droit anglais", 3, p. 372.

«L'Anglais du journalisme», 2, p. 272.

Larousse de la langue française en un volume, 1, p. 194.

Larousse en couleurs, 1, p. 96 .

«La tâche du traducteur», 4, p. 435.

LDS, 1, p. 54.

Législation, 1, p. 136.

"Les Fondements sociologiques de la traduction, 2, p. 267.

Lexicographie et terminologie juridique, 1, p. 148.

Lexicographie terminologique, 1, p. 156.

Lexico-sémantique, 2, p. 233.

Lexique (définition du), 2, p. 254

Lexique d'informatique, 1, p. 107.

Lexiques, 1, p. 85.

Lexis (le), 1, p. 93.

«Libre/littéral», (dichotomie), 4, p. 440.

Lieux suffixés en « $O$ » (à Paris), 3, p. 348

«Lift» (donner un), 3, p. 362.

Listes d'acquisitions (échange de), 1, p. 35.

«Listes de publications disponibles», 1, p. 53 .

«Littéral/libre» (dichotomie), 4, p. 440.

Littéralité, 4 , p. 436.

Littré (le), 1, p. 87.

Logique booléenne et logique pondérée, 1, p. 82 .

Logique pondérée et logique booléenne, 1, p. 82.

Luther, traducteur, 4, p. 432.

Lotte (D.S.), 1, p. 156.

Maintenance de la documentation, 1, p. 24.

Mammifères aquatiques (lexique A-F), 3, p. 325.

Manuel de l'utilisateur CAN/OLE, 1, p. 180

Manuel de rédaction des profils CAN/SDI, 1, p. 179.

Manuel du bibliothécaire documentaliste (le), 1, p. 84.

Marge et divergence, 4 , p. 453.

«Maxi-», 2, p. 251.

Mazout, 2, p. 263.

Médiatiseur, 3, p. 287.

Mémoire collective, 4, p. 441.

Meschonnic-Nida (querelle), 4, p. 436.

Messages, 3, p. 311.

Mets déshydratés, 3, p. 357.

Mets en conserve, 3 , p. 357 .

Mets surgelés, 3, p. 357.

Micro-traduction, 2, p. 239.

"Mimesis", 4, p. 438

"Mini ", 2, p. 249.

«Mini-», 2, p. 251.

MISTRAL, 1, p. 194.

Modes verbaux (traduction des divers), 3, p. 317.

Moteurs yolumétriques, 1, p. 105.

Mots-centaures, 3 , p. 346 .

Mots et termes, 3, p. 346.

Mots et termes, 3, p. 312. 
Mots nouveaux (composition par abrègement), 3, p. 345 .

Mots nouveaux (formation des), 3, p. 345.

Mots porte-manteau, 3, p. 346 .

"Mots sauvages» et suffixation en « $\mathrm{O}$ *, 3, p. 351 .

Mots-valises, 3, p. 346.

Nabokov, 4, p. 436

Nationalités suffixées en «O» (péj.), 3, p. 348.

Natis, 1, p. 49.

Néologie, 2, p. $280 ; 3,345$.

Néologie (l'acronymisation), 2, p. 250

Néologie (l'assimilation), 2, p. 250.

Néologie (la composition), 2, p. 250.

Néologie (la dérivation), 2, p. 250.

Néologie (la dérivation par suffixe), 2, p. 252.

Néologie (la formation directe), 2, p. 250.

Néologie (la formation indirecte), 2, p. 250.

Néologie (le changement de catégorie grammaticale), 2 , p. 250.

Néologie (l'emprunt), 2, p. 250

Néologie (le téléscopage), 2, p. 250.

Néologie (l'extension de sens), 2, p. 250

Néologie (sa dérivation régressive), 2, p. 250.

Néologie en français contemporain, 2, p. 249.

Néologie française, 1, p. 91

NEPAL (liste), 1, p. 53.

Nida-Meschonnic (querelle), 4, p. 436.

Nomenclature, 2, p. 254.

Nomenclature et terminologie, 1, p. 157.

Nomenclatures AFNOR, 1, p. 189.

Nonpareil, 1, p. 99.

NORIA, 1, p. 194.

NORIA accessible en ligne, 1, p. 194.

NORIANE, 1, p. 194.

Normalisation (définition de la), 1, p. 126

Normalisation (différents niveaux de), 1, p. 128.

Normalisation (documents de), 1, p. 126.

NORMalisation, $A$ utomatisation de la TERMinologie, 1, p. 192.

Normalisation d'entreprise, 1, p. 131.

Normalisation et recommandation (OLF), 2, p. 282.

Normalisation et recommandation terminologique, 1, p. 157.

Normalisation internationale, 1, p. 128.

Normalisation nationale, 1, p. 129

Normalisation régionale, 1, p. 129.

Normalisation technique et normalisation terminologique, 1, p. 126.

Normalisation terminologique, 2, p. 280 .

Normalisation terminologique et normalisation technique, 1, p. 126.

NORMATERM, 1, p. 132, 192.

Norme (définition du document appelé), 1, p. 126.

Norme (élaboration d'une), 1, p. 127.

Norme homologuée, 1, p. 128

Norme obligatoire, 1, p. 128.

Normes de l'Afnor, 1, p. 108

Normes de rendement du traducteur, 3, p. 383 .
Normes de vocabulaire et Normes techniques, 1, p. 127.

Normes du législateur, 1, p. 137.

NOrmes et Réglementation (Informations $A \mathrm{u}$ tomatisées), 1, p. 194.

Normes qualitatives du traducteur, 3, p. 383.

Normes techniques et normes de vocabulaire 1 , p. 127.

Nos, 3, p. 291.

«Notion» terminologique, 2, p. 256.

Nouveau Bescherelle, 1, p. 100.

Nouveau dictionnaire analogique du français, 1 , p. 97.

Nouveau dictionnaire des synonymes, 1, p. 97.

Nouveau dictionnaire étymologique et historique, 1, p. 97.

Nuances de la langue maternelle, 4, p. 391.

Numériser, 1, p. 105.

Numériseur, 1, p. 105

OACI, 1, p. 129.

Obligations du traducteur, 4 , p. 400

«Occasion» (avoir une occasion), 3, p. 363.

Office des normes du gouvernement canadien, 1, p. 56.

Omnis (1'), 1, p. 96.

On, 1, p. 197

ONGC, 1, p. 56 .

ONU (la Division de traduction), 1, p. 60

ONU (langues officielles à), 1, p. 58.

ONU (la Section de documentation et de terminologie), 1, p. 61 .

ONU (le Département des conférences), 1, p. 59.

ONU (recherche terminologique à l'), 1, p. 66.

Ordonnancement, 3, p. 355 .

Ordre alphabétique (classement par), 1, p. 17

Organisation arabe de normalisation et de métrologie, 1, p. 129.

Organisation de l'aviation civile internationale, 1, p. 129.

Organisation des Nations unies, 1, p. 58.

Organisation internationale de normalisation, 1, p. 56,128 .

Ortho Vert, Dictionnaire orthographique et grammatical, 1, p. 99.

Ouvrages lexicographiques, 1 , p. 164.

Ouvrages non lexicographiques, 1, p. 164.

Parole et langue en traduction, 4, p. 438.

Parataxe, 4, p. 434

Parigot, 4, p. 464

PASCAL, 1, p. 175.

Performance et compétence, 4, p. 438.

«Périodex », 1, p. 69

Périodique (résumé d'un texte de), 1, p. 75

Périodiques (catalogage des), 1, p. 73 .

Périodiques (choix des), 1, p. 72.

Périodiques (circulation des), 1, p. 24.

Périodiques et documents épars, 1, p. 68

Périodiques (indexation des), 1, p. 75 .

Périodiques (préparation matérielle des), 1 , p. 73 . 
Perspective, 4, p. 434.

Pertinence, 4, p. 391.

Petit Larousse illustré, 1, p. 96.

Petit Robert (le), 1, p. 93.

Planification, 3, p. 353.

Planification à court terme, 3 , p. 354 .

Planification à long terme, 3, p. 354.

«Planning (le), 3, p. 353.

«Planning d'approvisionnement, 3, p. 354.

"Planning de fabrication », 3, p. 354.

«Planning d'embauche, 3, p. 354.

«Planning d'étude du marché », 3, p. 354.

«Planning * financier $», 3$, p. 354.

Plat cuisiné, 3, p. 357.

PLATERM, 3, p. 287

PLATERM (vocabulaire), 3, p. 294.

PLATO, 3, p. 287.

PLATON, 3, p. 287.

Plats déshydratés, 3 , p. 357.

Plats en conserve, 3 , p. 357 .

Plats surgelés, 3, p. 357.

Pollution, 3, p. 359 .

Patinoire, 3, p. 385

«Période de voyage», 3, p. 273

Phraséologie et syntagmatique, 2, p. 231.

Pluralisme de l'usage et idiolecte, 4, p. 397.

Politisation de la traduction, 4, p. 443.

"Popular" (journal de la presse populaire), 2, p. 272.

Posséder les ressources de sa langue maternelle, 4 , p. 394

«Pouvoir de choc», 2, p. 273.

«Pouvoir-dire», 2, p. 268.

Pratique de la traduction, 4, p. 408.

Pratique et théorie, 4, p. 391.

Première conférence internationale sur les banques de terminologie, 1, p. 203.

Prénons suffixés en $" \mathrm{O} *, 3$, p. 348 .

Présentation de la nouvelle documentation, 1 , p. 24.

Présentoirs, 1, p. 24.

«Procédés de traduction $», 2$, p. 271.

Procédure, 1, p. 147.

Programmation, 3, p. 354.

Programmation de la production, 3, p. 354.

Programme appliqué à la sélection et à la compilation automatique de la littérature, 1, p. 175.

Psychanalyse et traduction, 4, p. 437, 442.

«Punch» (en journalisme), 2, p. 273.

Qualification (fr.), 4, p. 482.

Qualifications (angl.), 4, p. 480.

Qualification des emplois, 4, p. 482

Qualification du travail, 4 , p. 482 .

Qualification professionnelle, 4, p. 482.

Qualités souhaitées chez un traducteur, 2, p. 277.

Quillet (le), 1, p. 103.

Racines grecques et latines, 4, p. 492.

"Radar », 1, p. 69.

RADAR, 1, p. 175.
RADAR (répertoire), 1, p. 54.

Radiotéléphonique maritime (lexique A-F). 3, p. 364.

«Rahmenstruktur», 4, p. 437, 442.

Rapports terminologues-spécialistes, 3, p. 281.

Recherche bibliographique à moyen terme, 1 , p. 25.

Recherche terminologique à l'ONU, 1, p. 66 .

Recherche de l'équivalence, 2, p. 231.

Recherche documentaire biomédicale automatisée, 1, p. 181.

Recherche documentaire en traduction juridique, 1, p. 142.

Recommandation et normalisation (OLF), 2, p. 282.

Recommandation et normalisation terminologique, 1, p. 157.

Rédaction «bilingue», 4, p. 397.

Rédaction et traduction, 4, p. 397.

Rédaction française au Québec, 4, p. 398.

Rédaction parallèle, 4 , p. 397.

Rédaction unilingue, 4, p. 397.

Réductions, 1, p. 82.

REFCATSS, 1 , p. 54 .

Régime de retraite(s), 3, p. 385 .

Régionalismes, 2, p, 274.

Règlement technique, 1, p. 188.

Regroupement de l'information, 1, p. 16.

Regroupement et dégroupement, 1, p. 94.

Relations logiques à l'intérieur d'une paraphrase, 2, p. 240.

Relatives définitoires, 3 , p. 320.

Relatives non définitoires, 3, p. 320.

Repérage de l'information, 1 , p. 16.

Repérage et acquisition des documents, 1, p. 23.

Répertoire analytique des articles de revues du Québec, 1, p. 54, 69, 175.

Répertoire de l'Université Laval, 1, p. 129.

Réseau d'information et de documentation terminologique, 1, p. 199.

Résumé analytique d'un texte de périodique, 1 , p. 76 .

Résumé d'un texte de périodique 1, p. 75.

Résumé informatif d'un texte de périodique, 1 , p. 76.

Résumé signalétique d'un texte de périodique, 1, p. 76.

Ressources de la deuxième langue, 4, p. 394.

Révision (enseignement de la), 4, p. 396.

Robert, Paul, 4, p. 491.

Roudny, 1, p. 157.

«Roue de spécialité », 1, p. 16.

Roy (Alain), 1, p. 159.

SABINE' 1, p. 53, 174 .

Sager (J.C.), 1, p. 159.

Saint-Jérome, traducteur, 4, p. 431

Science terminologique, 1, p. 156.

Séance plénière du 31 mai 1980,3 , p. 384.

Section de documentation et de terminologie de 1'ONU, 1, p. 61.

Segmentation et commutation, 2 , p. 270.

Sémantique et compréhension, 2, p. 231. 
Sémantique et traduction, 2, p. 230.

Sémiotique des textes écrits, 3, p. 310

Sémiotique et terminologie, 3 , p. 313.

Sémiotique et traduction, 3, p. 310.

Sémiotique textuelle, 3 , p. 310 .

Sens du message, 4, p. 404.

Sens du dire, 4, p. 404.

Service de documentation (structure), 1, p. 15.

Service d'information technique, 1, p. 49.

Servitude en matière de traduction, 4, p. 447.

Servitude lexicale, 4, p. 450.

Sexe et suffixation en $« O », 3$, p. 352.

Signes vides, 4 , p. 408.

Signifiant et signifié en traduction, 4, p. 438.

Signifié et signifiant, 4, p. 438.

Signification du signe, 2, p. 269.

Signifié, 2, p. 268.

«Smart set», 2, p. 273.

«Société élégante», 2, p. 273.

Société québécoise d'information juridique, 1, p. 139.

Sociologie et traduction, 4 , p. 442 .

Sondage sur la traduction dans l'entreprise, 2, p. 276.

SOQUIJ, 1, p. $139,149$.

Spécialistes et terminologues, 2, p. 281.

Spécification technique, 1 , p. 188.

Soviétiques (terminologues), 1, p. 155.

Stages en traduction, 4, p. 493.

Stagiaires en traduction, 2, p. 276.

Statistiques dans le domaine de la néologie, 2 , p. 250.

STI, 1, p. 49

STQ (assemblée générale 1980), 3, p. 386.

STQ (reconnaissance professionnelle), 3, p. 385.

Structures de surface en traduction, 4, p. 438.

Structures profondes en traduction, 4, p. 438.

Suffixation parasitaire en $« \mathrm{O} », 3$, p. 347.

Suffixe $\ll O », 3$, p. 347 .

Supplément au Grand Larousse encyclopédique, 1, p. 105.

SVP documentation, 1, p. 53.

SVP militaire (Québec), 1, p. 56.

Syntaxe du français moderne, 1, p. 99.

Système Automatisé des Banques d'Information Non Numériques, 1, p. 53.

Système d'information bibliographique des Nations unies, 1, p. 64.

Système d'information documentaire des Nations unies, 1, p. 64

Système informatisé de banques d'information non numériques, 1 , p. 174

Système national d'information, 1, p. 49.

Système notionnel, 1, p. 155 .

Système PLATON, 3, p. 287.

Système d'ajustement, 4 , p. 469.

Systèmes juridiques (divers), 1, p. 135

Table ronde sur la traduction professionnelle, 3, p. 383.

«Télé-», 2, p. 251.

Temps verbaux (traduction des divers), 3 , p. 317.
Termes et mots, 3 , p. 312 .

Terminologie et sémiotique, 3, p. 313.

Tchécoslovaques (terminologues), 1, p. 157.

Techniques atraumatiques, 4 , p. 468.

Techniques de l'ingénieur 1, p. 102.

Techniques non-sanglantes, 4, p. 468.

Techniques sanglantes, 4 , p. 468 .

Techniques traumatiques, 4, p. 468.

Technor, 1, p. 104.

Terme technique (ce qu'est un), 4, p. 396.

Terminologie (but de la), 4, p. 467.

Terminologie (enseignement de la), 1, p. 161; $4,467$.

Terminologie (nouvelles acceptions du mot), 1, p. 196.

Terminologie (réflexion sur la), 2, p. 254.

Terminologie: art, pratique, ou science? (la), 1, p. 154.

Terminologie: discipline appliquée? (la), 1, p. 156.

Terminologie - la recherche, à l'ONU, 1, p. 66.

Terminologie et documentation, 1, p. 152.

Terminologie et lexicographie juridiques, 1, p. 148.

Terminologie et nomenclature, 1, p. 157.

Terminologielehre, 1, p. 156.

Terminologues (perfectionnement des), 1, p. 199.

Terminologues britanniques, 1, p. 159.

Terminologues et spécialistes, 2 , p. 281.

Terminologues français, 1, p. 159.

Terminologues germano-autrichiens, 1, p. 155.

Terminologues soviétiques, $1, \mathrm{p} .155$.

Terminologues tchécoslovaques, 1, p. 157.

Terminoq II (système), 1, p. 14, 182.

Terminoq 2, 1, p. 182.

TermNet Newsletter, 1, p. 201.

TEXTILDOQ, 1, p. 175.

«thèque», 2, p. 251.

Théorie et doctrine de la traduction, 4, p. 402.

Théorie et pratique de la traduction, 4, p. 391.

Théorie inspirée de la pratique, 4, p. 401.

Théorie interprétative de la traduction, 4, p. 404.

Thermaliser, 1, p. 105.

Thesaurus (définition du), 1, p. 78 .

Titres d'ouvrage ou de films (traduction de), 4 , p. 442.

Traducteur (obligations du), 4, p. 400.

Traducteur dépositaire, 4 , p. 441.

Traducteur/lecteur (couple), 4, p. 440

Traducteur médiateur, 4, p. 441 .

Traducteurs et écrivains, 4, p. 396.

Traduction (histoire de la), 4, p. 430.

Traduction (théorie et pratique de la), 4, p. 391

Traduction dans l'entreprise (la), 2, p. 276.

Traduction et rédaction, 4 , p. 397.

Traduction et sémantique, 2 , p. 230.

Traduction et sémiotique, 3, p. 310 .

Traduction et transcodage, 2 , p. 268.

Traduction juridique (recherche documentaire en), 1, p. 142.

Traduction juridique, 3 , p. 316 . 
Traduction littéraliste, 4, p. 431.

Traductions parallèles, 4, p. 453

Traductologie, 2, p. 270.

Traduire, c'est..., 4, p. 435.

"Traduit du» (le), 4, p. 444.

Traité de la formation de la langue (le), 1, p. 88

Traitement des documents, 1, p. 85.

Transcodage et traduction, e, p. 268.

Trésor de la langue française (le), 1, p. 91

Troisième colloque OLF-STQ, 2, p. 278.

Troncations dans la langue populaire, 3 , p. 347.

UNBIS, 1, p. 64

UNDIS, 1 , p. 64

UNDOC, 1, p. 64.

UNIBID, 1, p. 204.

UNION, 1, p. 181.
Unitès de traduction, 2, p. 270

Unités terminologiques, 3, p. 314 .

Univocité du terme, 1, p. 155.

URBADOQ, $1, \mathrm{p}, 175$

Usager des traductions, 4, p. 399.

UTLAS, 1, p. 51.

VGOS, 1, p. 150 .

VINOKUR, 1, p. 155.

Vocabulaire AFNOR, 1, p. 189.

Vocabulaire (définition du), 2, p. 254.

Vocabulaire PLATERM, 3, p. 294.

Vulgate (la), 4, p. 431.

WERSIG (G.), 1, p. 155.

Wüster (E.), 1. p. 155, 213. 